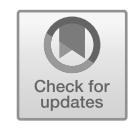

\title{
6 Die agential cuts dieser Arbeit
}

Mit dieser Arbeit versuche ich zu zeigen, dass mit dem Agential Realism als wissenschaftstheoretische Position eine queer(end)e Experimentalpsychologie möglich ist, die ihr queer(end)es Potential nicht durch ihre Themenwahl, sondern durch ihre grundlegende Herangehensweise an Phänomene erhält. Wie also legt man ein Experiment an, das auf Dann-Gegebenheiten einer Entität schließen will, während man gleichzeitig eine Differenz als Resultat dessen erachtet, wie Mächtigere eine historische Situation interpretieren (vgl. Einleitung S. 3)? - Als Antwort schlage ich vor, diese mächtige Position aktiv einzunehmen und möglichst verantwortungsvoll damit umzugehen, dass Forschende mitunter die Differenz-Herstellenden oder Differenz-Wiederholenden sind. Dieses möglichst verantwortungsvoll sehe ich als die große Herausforderung, die den Einbezug von in klassischem Realismus bisher als unwissenschaftlich geltenden Werten benötigt. Auch mit experimentalpsychologischen Differenz-Herstellungen können interessante situative Zusammenwirkungen und somit situierte Möglichkeitsräume beschrieben werden, die anzeigen, welche Realisierungsmöglichkeiten eine spezifische Konfiguration zulässt. Ebenso können Realisierungsräume beschrieben werden, die anzeigen, um welche Werte die Realisierungen pendeln. Eine weitere Herausforderung für ARqE sehe ich darin, was als interessant gilt, wenn nicht mehr Forschungsgrund sein kann, etwas über Gegebenheiten der Welt zu konstatieren, sondern immer nur situiertes Wissen geäußert werden kann. Zunächst könnte jeder Möglichkeitsraum als interessant gelten. Wenn aber prinzipiell ethische Überlegungen jede cut-Setzung lenken sollen, bei der Forschende eine Differenz herstellen oder wiederholen, sieht man sich mit fast jedem Experiment mit Fragen konfrontiert, die zahlreiche Forschende auch schon heute kennen. Ein Beispiel hierfür wäre die Forschung mit menschlichen Stammzellen, für deren Studiendesigns nicht nur die technische Machbarkeit entscheidend ist, sondern auch ethische Entscheidungen einer Gesellschaft eine große Rolle spielen. Aus solchen Debatten kann vermutlich viel über die praktische Umsetzbarkeit gelernt werden - beispielsweise die Sinnhaftigkeit und Einsetzbarkeit von Ethikkommissionen. Wenn einzelne Entschei- 
dungen eines Forschungsprozesses - z. B. aufgrund geringer(er) Tragweite leichter zu fällen sind, könnte dies ein Grund sein, der sich auf die praktischen Umsetzungen auswirkt, aber nicht ethische Überlegungen obsolet macht. Mit Blick auf die Mitwirkungen von Forschenden werden u. U. zahlreiche Konfigurationen deutlich, in denen Forschende am Machterhalt dominanter Gruppen beteiligt waren bzw. es immer noch sind, was aus queertheoretischer Perspektive zu ändern ist.

In zahlreichen Konfigurationen mögen die Mitwirkungen von experimentalpsychologischen Messungen vernachlässigbar sein. Dies kann jedoch nicht vorausgesetzt werden und es stellt damit eine weitere Herausforderung für queer(end)e Experimentalpsycholog_inn_en dar, dies erkennen zu können. Zudem kann sich die Feststellung von ist zu vernachlässigen auch nur auf ein relativ zu $X$ beziehen. So sehr wie es dafür, dass ich meinen Sessel im Raum finde, $\mathrm{zu}$ vernachlässigen ist, dass das Lichteinschalten meine Möbel verrückt, so wenig ist dies bei der genauen Positionsmessung eines Teilchens zu vernachlässigen. Das Urteil, dass es zu vernachlässigen wäre, dass per Versuchs- und Messanordnung ein Teil von Studienpartner_inne_n zu Schnellergehenden und ein anderen Teil zu Langsamergehenden gemacht werden (zum Beispiel bei Bargh, Chen \& Burrows, 1996), macht nur in Relation zu einem weiteren Wert Sinn, beispielsweise dass diese Kategorisierung keine weiteren Auswirkungen habe. Das heißt für ARqE steht auch viel Forschung über relative Konsequenzen von situierten Forschungssettings an. Dies ersetzt keinesfalls ethische Abwägungen. Es informiert ethische Abwägungen viel eher, wenn der Möglichkeitsraum und der Realisierungsraum von Konsequenzen eines bestimmten Effektes besser beschrieben werden können. Wenn eine bestimmte, aufgrund bestimmter Werte unerwünschte, Realisierung in einem konkreten Setting als sehr unwahrscheinlich anzunehmen ist, dann kann dies als Begründung verwendet werden, dass in diesem Fall die Differenzherstellung durch die Forschende zu vernachlässigen ist. Doch auch dies wäre eine situierte Entscheidung und nichts, was in der Natur der Sache selbst läge.

Eingangs habe ich die Frage aufgeworfen, wie in einem Experiment eine Quantifizierung von Gegebenheiten und gleichzeitig deren Dekonstruktion 
angestrebt werden kann. Dieselben relata sind nur zu dekonstruieren, wenn trotz - oder eventuell sogar aufgrund - ihrer Quantifizierung ihre Bedingungsabhängigkeit deutlich wird. Wenn beispielsweise die Anzahl und die inhaltlichen Möglichkeiten von momentaner geschlechtlicher Selbstkategorisierung für einen spezifischen Kontext abgewogen werden, kann es gelingen, deren Situiertheit zu verdeutlichen und trotzdem mit einer Auswahl eine Quantifizierung zu vollziehen, die gleichzeitig die Inhärenz- und Stabilitätsannahmen von Geschlechtsidentität dekonstruiert. Einfacher ist vermutlich, bei Quantifizierung der einen relata andere relata zu dekonstruieren, was ich - je nach relata, um die es sich handelt - immer noch als queer(end)e Handlung im Sinne Butlers erachten würde, da auch queertheoretisch keine gänzliche Kategorienlosigkeit angestrebt wird, sondern eine Verschiebung hin zu größerer Chancengleichheit (vgl. Kapitel 2.3). Wiederum kann es auch nur situativ im Einzelfall, relativ zu einem bestimmten Wert, beurteilt werden, ob dieses spezifische Quantifizieren der einen relata bei gleichzeitiger Dekonstruktion anderer relata eine queer(end)e Handlung ist. Wenn ich es für akzeptabel halte, ein Experiment als queer(end) zu beurteilen, auch wenn bestimmte ralata durch ihre Konfigurationen nur quantifiziert und nicht dekonstruiert werden, macht dies wieder die Relativität jeden Urteils und die Unausweichlichkeit von Diskussionen über solche Einschätzungen deutlich. Eine ARqE-Forschungsgemeinschaft müsste sich diesen Herausforderungen in Zukunft stellen.

Wenn ich behaupte, dass mit Barads Agential Realism eine queer(end)e Experimentalpsychologie möglich ist, befindet sich meine Argumentation selbst in Konfigurationen und hängt vom situierten Apparatus of Knowledge (im Sinne Butlers wie Barads) ab. Ich spreche beispielsweise über Experimente als wissenproduzierende, die mit spezifischen Voraussetzugen arbeiten (Kapitel 3). Anschließend spreche ich über alternative Voraussetzungen für ein alternatives experimentelles Handeln (Kapitel 4), welches dennoch in bestimmte Verständnisweisen eingebunden ist. So ist jedes einzelne Konzept innerhalb meiner Aussagen verwoben in Lesarten, Praktiken und situierten Möglichkeitsraum. Damit referenziert die Arbeit auf bestimmte Diskurse (z. B. die einer akademischen Experimentalpsychologie bzw. Queer Theory bestimmter Länder), beteiligt sich an der Diskussion, an der bestimmte Menschen beteiligt und bestimmte andere 
Stimmen nicht beteiligt sind und will dabei ein weiterführender Diskussionsbeitrag sein. Weiterhin erzeugt meine Argumentation selbst differenzierende cuts. Konzepte, mit denen ich arbeite - z. B. Queer Theory, Agential Realism oder Experimentalpsychologie-, stelle ich selbst situiert mit her bzw. wiederhole deren Herstellung. Durch meine Formulierungen trenne ich manche Konzepte als geschlossen heraus, während andere als Vermischtes dargestellt werden. Im Folgenden werfe ich einen Blick auf die Relationen dieser herausgeschnittenen Figuren untereinander und berücksichtige dabei folgende Verhältnisse Agential Realism zu Queer Theory, wissenschaftstheoretische Position zu Methodenwahl, Experimentalpsychologie mit klassischem Realismus zu Experimentalpsychologie mit AR.

Agential Realism : Queer Theory

Die queertheoretischen Eingangszitate dieser Arbeit (von Wittig und Butler, vgl. S. 1) habe ich bereits so ausgewählt, um später eine gewisse Nähe des Agential Realism zu queertheoretischen Positionen herzustellen bzw. zu wiederholen. Kapitel 4.3 versucht, die Queerness der ARqE (über ihre Selbstbezeichnung als solche hinaus) zu zeigen. Barads eigene Schnitte, zwischen Agential Realism und Queer Theory einmal eine Grenze zu ziehen und einmal nicht, erscheinen mir situativ. Einerseits benennt sie die „Performativität der Natur“ als queer (Barad, 2012, Natures Queer Performativity) und präsentiert den Agential Realism als Grundhaltung, mit der die Queerness der Welt - im Gegensatz zu repräsentationalistischen Grundhaltungen - leicht lesbar wird. Weitere Beispiele von radikal queeren Konfigurationen diskutiert sie u.a. in Transmaterialities (Barad, 2015). Andererseits formuliert sie klare Abgrenzungen, wo ihr der Agential Realism über queertheoretische Positionen hinauszugehen scheint. $\mathrm{Zu}$ mindest im Jahr 2008 erachtet(e) sie beispielsweise noch als nötig: "a radical rethinking of the nature of experience (for example, touch and vision), of theory, and their interrelationship, as well as many other core concepts that are still taken for granted by queer theorists" (Barad, 2008, S. 336). In Barads aktuellem Hauptwerk Meeting the Universe Halfway (2007) finden sich zahlreiche Abgrenzungen gegenüber Butlers Positionen. Barad bezeichnet Butlers Positionen dabei 
nicht als grundverschieden vom Agential Realism, aber als im Vergleich zu diesem verkürzt und "limited to the production of human bodies (and only certain aspects of their production, at that)" (Barad, 2007, S. 145). Diese Einschätzungen teile ich nicht, sondern sehe Barads Abgrenzung an dieser Stelle wie die einer Botanikerin, die - über Botanik hinaus - eine Theorie der Verbundenheit der Lebensformen entworfen hat (aber über diese mit Referenzierung auf Botanik spricht) und nun einer Zoologin, die - über Zoologie hinaus - ebenfalls eine Theorie der Verbundenheit der Lebensformen entwickelt hat (aber über diese mit Referenzierung auf Zoologie spricht), vorwirft, nur über Zoologie zu sprechen. Meines Erachtens entwirft auch die Queer Theory eine Perspektive auf ontologische, epistemologische und erst recht ethische Fragen unserer Welt. Diese sind jedoch nur sehr selten in einen empirischen Diskurs eingebettet. Bei ethicoepistemontologischen Fragen in queertheoretischen Analysen standen höchstens zu Anfang Geschlechts- und Sexualitätskategorien im Fokus. Sofort wurden diese in Verschränkung mit weiteren Strukturierungskategorien wie Ethnizität, Klasse, Alter und Fähigkeiten betrachtet. Insgesamt geht es um die Grundhaltung gegenüber Wissen und Handeln in der Welt. Noch immer geht es in queeren Analysen darum zu erkennen, welche Effekte dadurch erzeugt werden, wie wer über wen spricht, wie wer gegenüber wem handelt und wie negative Auswirkungen dessen reduziert bis verhindert werden können. Bee Scherer beschreibt:

[Q]ueer interventions can be understood as countering the psychosocial violence against queer subjects; these are done through concrete acts of resistance in the public sphere through activism, art(ivism); political and civic disobedience and protest; through individual, contextual modes of resistance, counter-scripts and resilience. (Scherer, 2016, S. 1-2)

So gehen die Einsatzgebiete eines queeren(den) Denkens weit über klassische wissenschaftliche Interessen hinaus. Im Fokus queeren(den) Denkens steht die Kritik an einem Handeln, das auf als standpunktlos angesehenem Wissen beruht. Theoretiker_innen wie Aktivist_inn_en versuchen Schritt für Schritt aufzuzeigen, dass dieses sogenannte Wissen tatsächlich werte- und voraussetzungsgebunden ist. Dies zeigt meines Erachtens das wissenschaftstheoretische 
Interesse der Queertheorien, auch wenn bisher seltener auf empirische Forschung geblickt wurde, doch die queer(end)e Kritik kann und soll in allen Feldern menschlicher Betätigung geübt werden und ganz besonders in allen wissenschaffenden Disziplinen und allen Methoden. Sullivan und Murray (2009) formulieren als queer(end)es Ziel die Destabilisierung des Selbstverständlichen: "Queer, at least as we understand it, is a heterogeneous and multidisciplinary practice aimed at 'bringing forth' and thus denaturalising the taken for granted, the invisiblised, the normalised; in short, the dispotitifs or technés of (necessarily material) (un)becoming." (Sullivan \& Murray, 2009, S. 4) Solche queertheoretischen Analysen zeigen das Gemachtsein von (sozialen) Kategorien auf, wie Barads Analysen die Bedingungsabhängigkeit materieller Entitäten aufzeigen. Der Großteil der bisherigen queertheoretischen Analysen mag sich darauf beziehen, was wir als menschlichen Bereich abgrenzen könnten. Queertheoretische Intention ist es jedoch gerade, solche Abgrenzungen zu dekonstruieren. In ihrer Einleitung zu Queering the Non/Human schreiben Giffney und Hird (2008, S. 5): "Queer is employed here as a collection of methodologies to unpick binaries and reread gaps, silences and in-between spaces." Nach meiner Einschätzung nimmt Butler ebensowenig eine Begrenzung auf einen sogenannten menschlichen Bereich vor. In Körper von Gewicht schreibt sie:

Was ich an Stelle dieser Konzeptionen von Konstruktion vorschlagen möchte, ist eine Rückkehr zum Begriff der Materie, jedoch nicht als Ort oder Oberfläche vorgestellt, sondern als ein Prozeß $\beta$ der Materialisierung, der im Laufe der Zeit stabil wird, so daß sich die Wirkung von Begrenzung, Festigkeit und Oberfläche herstellt, den wir Materie nennen. Daß Materie immer etwas zu Materie Gewordenes ist, muß meiner Meinung nach mit Bezug auf die produktiven und eben auch materialisierenden Effekte von regulierender Macht im Foucaultschen Sinne gedacht werden. Dementsprechend lautet die Frage künftig nicht mehr, wie das soziale Geschlecht als eine und durch eine bestimmte Interpretation des biologischen Geschlechts konstituiert wird (eine Frage, bei der die ,Materie' des biologischen Geschlechts von der Theorie ausgespart bleibt), sondern vielmehr: Durch welche regulierenden Normen wird das biologische Geschlecht selbst materialisiert? Und wie erklärt sich, daß die Behandlung der Materialität des biologischen Geschlechts als 
eines Gegebenen die normativen Bedingungen für dessen Auftreten voraussetzt und konsolidiert? (Butler, 1993/1997, S. 32)

Butler interessiert sich sowohl für Materie, wie für nicht-materielle Phänomene, auch ohne dazwischen eine Grenze zu ziehen, sondern gerade in deren Verschränkung. Es scheint mir lediglich so, dass solche Fragen wie „Durch welche regulierenden Normen wird das biologische Geschlecht selbst materialisiert?" (siehe Zitat von eben, Butler, 1993/1997, S. 3) in Folge ihrer Publikation detaillierter von anderen Theoretiker_inn_en (z.B. Voss, 2011, 2013), ausgeführt wurden. Dies bedeutet aber nicht, dass Butler nicht das Gewordensein von dann-materiellen Strukturen mitdenken würde.

Entsprechend erachte ich den Agential Realism nicht als eine Grundhaltung, die über Queertheorien hinaus geht, sondern als eine, die eine Brücke schlägt, an der queere Kritiken bisher lediglich wenig interessiert waren: Sie schlägt diese Brücke damit, eine Ausformulierung der wissenschaftstheoretischen Position zu liefern, mit der quantitative Experimente möglich sind, um anschließend Aussagen über situierte Phänomene treffen zu können. Queertheorien haben stark auf die Dekonstruktion Machtgefälle erhaltenden Wissens fokussiert und wenig Interesse an der Formulierung von sogenannten Tatsachen und seien es lokale, temporäre - gezeigt. Ich halte die queertheoretisch informierte Formulierung von situiertem Wissen für lohnenswert. Ebenso ist die anzuwendende Methode für das Gewinnen einer situierten Einsicht situativ auszuwählen, was zu dem interessanten Verhältnis von wissenschaftstheoretischer Position zu Methodenwahl führt.

\section{Wissenschaftstheoretische Position : Methodenwahl}

Neben den vielen quantitativ Forschenden mit klassisch realistischer Position gibt es zahlreiche Forscher_innen, die metatheoretisch von klassischem Realismus ausgehen und ein spezifisches Phänomen qualitativ erfoschen. Ebenso sind Forschende denkbar, die nicht von klassischem Realismus ausgehen und dennoch quantitative Methoden verwenden. So kann ich die Methodenwahl, ob beispielsweise qualitativ oder quantitativ vorgegangen wird, als orthogonal zur 
metatheoretischen Position, ob beispielsweise klassischer Realismus angenommen wird oder nicht, begreifen. Das eine müsste nicht mit dem anderen zusammenhängen. Entsprechend wird oft von gegenstandsangemessenen und nicht von wissenschaftstheorieabhängigen Methoden gesprochen. Praktisch scheint es aber dennoch eine Korrelation (in der europäisch und US-amerikanisch geprägten Psychologie) derart zu geben, dass quantitatives Vorgehen aktuell fast ausschließlich vom klassischen Realismus ausgeht (vgl. z. B. Kapitel 3.1). Selbstverständlich ist der Agential Realism nicht die einzige alternative wissenschaftstheoretische Position, die quantitatives Vorgehen erlaubt. Ich habe in dieser Arbeit auf den Agential Realism fokussiert und angestrebt zu zeigen, dass die Suche nach Zusammenwirkungen auch ohne klassisch realistische Position funktioniert und Sinn macht; dies jedoch nicht als Hintergrund für die Suche nach Wahrheit (vgl. Popper, 2002, siehe Kapitel 2.1.1, S. 79), sondern für Einsichten über situierte Möglichkeits- und Realisierungsräume. Wer eine queerere Welt anstrebt und trotzdem quantitativ Zusammenwirkungen beschreiben will, ist meines Erachtens nicht länger in einem Widerspruch gefangen, sondern kann mit Agential Realism eine queer(end)e Experimentalpsychologie betreiben. Das soll nicht bedeuten, dass dies immer die passendere Kombination für alle ist, die queer(end) psychologisch forschen wollen. Vielerlei Aussagen sind sicher immer noch besser durch qualitative Methoden zu erzielen. Die Frage, wie sich Agential Realism für qualitative Methoden eignet, habe ich bisher nicht behandelt. Mein Interesse galt zunächst der Konfrontation und Dekonstruktion der Widersprüchlichkeiten von queeren Kritiken und klassischem experimentalpsychologischem Vorgehen. Ich gehe davon aus, dass die wissenschaftstheoretische Position nicht direkt die auszuwählende Methode vorgibt. Dennoch beinhaltet sie erkenntnistheoretische Axiome, die beispielsweise vorgeben, wie mit Schwierigkeiten wie dem Einfluss durch die Messung umzugehen ist. Wenn man klassisch realistisch von bedingungslos bestehenden stabilen Entitäten ausgeht, macht es Sinn, nach deren Beschaffenheit zu fragen, Einflüsse der Messung als Störgröße zu behandeln und entsprechend zu versuchen, diese Einflüsse zu minimieren. Methoden wiederum bauen auf gewisse Voraussetzungen auf. Daher mag die Methodenwahl indirekt doch durch die konkreten erkenntnistheoretischen Annahmen einer wissenschaftstheoretischen Position (z. B. Entitätenrealismus) mitbestimmt sein. 
Bezüglich eines $t$-Tests habe ich in dieser Arbeit diskutiert, dass dieser auch aus einer AR-Position heraus anwendbar ist. Anders als aus der klassisch realistischen Position wird jedoch kein zufälliger Messfehler angenommen. Vielmehr wird davon ausgegangen, dass das Pendeln von Werten den Realisierungsraum für eine Konstellation anzeigt. Nichtdestotrotz steht für viele etablierte psychologische quantitative Methoden noch die detallierte Prüfung ihrer Voraussetzungen für eine Kompatibilitätseinschätzung bezüglich Agential Realism aus. Dies ist ebenfalls eine der zukünftigen Herausforderungen für $\mathrm{ARqE}$, worauf ich weiter unten zurückkomme. Zuvor soll der angesprochene Unterschied zwischen klassischem Realismus und Agential Realism in der Herangehensweise an erkenntnistheoretische Probleme erneut kurz beleuchtet werden.

Experimentalpsychologie mit klassischem Realismus : Experimentalpsychologie mit Agential Realism

Einige queertheoretische Kritiken an Experimentalpsychologie mit klassischem Realismus - zum Beispiel die Erinnerung an Kontextabhängigkeit eines Phänomens - können von dieser verstanden und anerkannt werden. Im Umgang mit dieser Kritik zeigt sich jedoch eine grundlegend unterschiedliche Herangehensweise von klassischem Realismus und Agential Realism. Der AR inkorporiert die Einsicht, dass Kontext eine Rolle spielt, in die Idee vom Erkenntnisgewinn. Die klassisch realistisch arbeitende Experimentalpsychologie geht dagegen davon aus, dass Kontexteinflüsse prinzipiell quantifizier- und berücksichtigbar sind und geht entsprechend weiterhin davon aus, einen wahren Effekt finden zu können (vgl. Kapitel 3). Ich möchte dies an der Diskussion von zwei Artikeln von Klaus Fiedler verdeutlichen - die nebenbei bemerkt sogar weiter als gewöhnlich in Richtung einer agential realist Perspektive gehen.

Fiedler (2011) greift in seinem Artikel "Voodoo Correlations are Everywhere - Not Only in Neuroscience" eine Feststellung von Vul, Harris, Winkielman und Pashler (2009) auf, die ein Vorgehen in den Neurowissenschaften kritisieren, um deren grundsätzliche Kritik auch für psychologische Forschung $\mathrm{zu}$ diskutieren. Inhaltlich geht es darum, dass durch gewisse 
Entscheidungen im Studiendesign Korrelationen vergrößert werden können. Fiedler (2011) kann das von Vul et al. (2009) kritisierte Vorgehen in die Psychologie übertragen und zudem zahlreiche weitere Beispiele nennen, in denen Entscheidungen der Forschenden das Ergebnis mitbestimmen: "[I]ndependence can be lost in many other arbitrary sampling decisions in the research process, such as the selection and publication of research questions and the operationalization of variables, tasks, stimuli, and instructions." (Fiedler, 2011, S. 164) Er diskutiert Einflüsse in Form von: "biases from the study design", "biases from selecting variables and measures", "biases from the analyses" und "biases from selective correction, publication, and funding of research". Nach Vorlegen zahlreicher Einflussfaktoren gilt sein Interesse dann folgender Frage: "What remedies or countermeasures may avoid or reduce those biases?" (S. 168) Seine Reaktion auf die Einsicht, dass eine Vielzahl von Entscheidungen im Forschungsprozess das Ergebnis mitbestimmen, besteht also in dem Versuch, solche Verzerrungen (biases) zu vermeiden oder wenigstens zu reduzieren. Damit wird weiter das Ideal von "unbiased methodologies" (Fiedler, 2011, S. 169) verfolgt.

In seinem Artikel "From intrapsychic to ecological theories in social psychology: Outlines of a functional theory approach" kann Fiedler (2014) ebenfalls zahlreiche empirische Beispiele dafür anführen, dass der weitere Kontext auf die Ergebnisse wirkt. Er differenziert diesbezüglich zwischen intrapsychic processes und extrapsychic conditions. Seine Diagnose ist (wie auch meine eigene, vgl. Kapitel 3), dass letztere in psychologischer Forschung kaum beachtet werden: "There is little interest in such extrapsychic conditions (...) Social psychologists exhibit little interest in such genuinely social conditions (...) They not even seem to propagate a conceptual framework that gives meaning to environmental variables." (Fiedler, 2014, S. 666) Er plädiert dafür, diese extrapsychischen Bedingungen in Form eines environmental approach in Zukunft besser zu beachten, und ist mit dieser Forderung nahe an Positionen des Agential Realism und der Queer Theory. Doch letztlich betrachtet er die Beachtung extrapsychischer Bedingungen als Strategie, um sogenannte explanatorische Distanz zu vergrößern ("How to increase explanatory distance", S. 659): 
[E]xplanatory concepts have to be sufficiently distant and detached from the phenomena they are to explain. Moreover, theoretical concepts have to appear 'objective' in the sense that they can be measured independently of the dependent measures meant to capture the phenomena to be explained. (Fiedler, 2014, S. 658)

Fiedler spricht sich also deshalb für die Beachtung von extrapsychischen Bedingungen als mitverantwortlich für das Ergebnis aus, um dadurch die Objektivität und die Distanz zwischen Explanans und Explanandum zu steigern. Dies hält er vor allem deshalb für erstrebenswert, weil er davon ausgeht, dass Objektivität und jene Distanz zentrale Bestandteile von wissenschaftlichem Fortschritt seien: "In other words, theoretical distance and objectivity are not just useful for the public image; they are key properties of scientific growth." (Fiedler, 2014, S. 659)

Fiedler geht es also nicht nur um die Vermeidung von Zirkularität, sondern vor allem um eine sogenannte objektivierende Trennung der erklärenden theoretischen Konzepte von dem gemessenen Ergebnis. Dies widerspricht dem zentralen Verständnis des Agential Realism, dass die Messweise - also die agencies of observation - und damit auch die theoretischen Konzepte das gemessene Ergebnis mitbestimmen und ohne letzere auch gar keine Messung möglich wäre. Laut Barad setzen ja gerade erst die Konzepte, mit denen an eine Messung herangegangen wird, jene agential cuts, die die dann-bestimmten relata situativ aus ihren relations herausschneiden. So kann aggressives Verhalten nicht ohne Annahmen und Setzungen über das Konzept Aggression beschrieben werden. Gleiches gilt für eine Kausalrelation mit dem Resultat aggressives Verhalten, die nicht ohne Annahmen und Setzungen über auslösende Komponenten beschrieben werden kann.

Fiedlers Vorgehensweise zeigt ein gewisses Maß an Selbstanwendung der psychologisch wohl unumstrittenen Einsicht, dass sich Menschen unter verschiedenen Bedingungen mitunter verschieden verhalten und fühlen. Entsprechend plädiert er für Umwelt-Theorien, folgt dabei jedoch weiter dem Entitätenrealismus, dass menschliches Verhalten etwas ist, was - zumindest theoretisch - objektiv beschrieben werden könnte. Seiner Ansicht nach stehen 
dieser Beschreibung lediglich epistemologische Probleme bzgl. Objektivität entgegen, weshalb es seiner Meinung nach sinnvoll wäre, sogenannte Verzerrungen aufzuspüren und zu reduzieren.

Hegarty und Bruckmüller (2013) gehen mit der Einsicht über nonneutrale Beschreibungen und Erklärungen anders um und liefern damit eine Anwendung für die Anerkennung von relata als within-relations. Sie beschreiben das Phänomen, dass ein Vergleich nicht formuliert werden kann, ohne das eine Vergleichsobjekt als Figur und das andere als Hintergrund zu positionieren (zumindest in den Sprachen des europäisch und US-amerikanisch geprägten Raumes). Im Beispiel „Männer haben mehr Einkommen als Frauen“ sind Männer die Figur von dem Hintergrund Frauen, während es bei „Frauen haben weniger Einkommen als Männer" umgekehrt ist. Logisch wird in beiden Fällen dasselbe gesagt. Eine unterschiedliche Anordnung von Figur und Hintergrund hat jedoch Auswirkungen auf gemessenen Einschätzungen. Dies gilt für sinnlose Silben (wie "ZUM is near GAX", vgl. Gleitman, Gleitman, Miller \& Ostrin, 1996; zit. n. Hegarty \& Bruckmüller, 2013) und für menschliche Gruppen: Der als Hintergrund positionierten Gruppe wird jeweils höherer Status und mehr Macht zugesprochen (vgl. Bruckmüller \& Abele, 2010; zit. n. Hegarty \& Bruckmüller, 2013). Dies ist für kulturelle Bereiche relevant, in denen sich eine bestimmte Gruppe als Hintergrund etabliert hat. So sind beispielsweise auf der Führungsetage der Wirtschaft Männer die Norm bzw. der Hintergrund und Frauen die (dann-erklärungsbedürftige) Figur. Vergleiche werden typischerweise so formuliert, dass sie diese Konfigurationen widerspiegeln: Im aktuellen Beispiel würden Frauen als Figur positioniert und artikuliert ,wie Frauen sich von Männern unterscheiden“. Dadurch werden Status- und Machtunterschiede als größer und legitimer erachtet und vermehrt statusbezogene Geschlechterstereotype zugeschrieben, als wenn der Vergleich umgekehrt formuliert wird (,wie Männer sich von Frauen unterscheiden“; Hegarty \& Bruckmüller, 2013).

In der Beschreibung dieser empirischen Ergebnisse vollziehen Hegarty und Bruckmüller mehrere ARqE-Schritte. Zum einen lokalisieren sie die Ursachen für gefundene Asymmetrien nicht allein in der kognitiven Mechanik der Handelnden, sondern erachten diese als "resulting from history, communication 
pragmatics, learning and knowledge activation" (Hegarty \& Bruckmüller, 2013, S. 177). Damit wenden Sie schon die von Fiedler geforderte Beachtung der extrapsychischen Bedingungen an.

Darüber hinaus wenden sie das Foucault'sche Machtverständnis von Disziplinarmacht an. Demnach übt nicht eine mächtige Person sichtbar Macht über statusniedrigere Personen (Disziplinierte) aus. Vielmehr werden die Disziplinierten sichtbar gemacht, wodurch es für Institutionen leichter wird, mit diesen Individuen in einer Weise umzugehen, die objektiv, rational und fair erscheint, obwohl sie einem Standpunkt angehört. Hegarty und Bruckmüller (2013) lesen die Auswirkungen von asymmetrischen Beschreibungen und Erklärungen von Gruppenunterschieden als Realisierung von Disziplinarmacht, weil die als Figur exponierte Gruppe durch die Sichtbarmachung diszipliniert wird und Auswirkungen erfährt. Wichtig ist, dass dies nicht nur in ihrem Experiment geschieht, sondern auch dann, wenn sogenannte Laien einen Vergleich formulieren und erst recht wenn sich in einer Kultur in bestimmten Bereichen eine bestimmte Konstellation (wie Männer als Norm) etabliert hat. Die Autor_inn_en beschreiben außerdem die asymmetrischen Formulierungen (inklusive Auswirkungen) von Forschenden und betreiben damit die vom AR geforderte Selbstanwendung, Einflüsse nicht nur bei ihren Studienpartner_innen zu erkennen, sondern bei allen Handelnden/Sprechenden. Ihr Beispiel führt außerdem vor, dass es wie im Foucault'schen Verständnis kein Außerhalb von Machtrelationen gibt. Es ist unmöglich, eine vergleichende Aussage neutral oder symmetrisch zu treffen. Selbst wenn beide Formulierungen genannt würden, würde eine zuerst genannt, was wieder asymmetrische Auswirkungen hat. Es macht also keinen Sinn zu versuchen, die Asymmetrie nicht zu begehen. Vielmehr müsste man aktiv anders damit umgehen, wenn man die Auswirkungen problematisch findet. Die Arbeit von Hegarty \& Bruckmüller (2013) legt zum Beispiel nahe, solche Formulierungen $\mathrm{u}$. U. bewusst untypisch zu formulieren, wenn man beispielsweise der Etablierung einer Gruppe als Norm entgegenwirken möchte. Die Autor_inn_en fordern mehr Arbeit $\mathrm{zu}$ diesen Einsichten, unerwünschte Auswirkungen beeinflussen zu können, doch nennen sie die Asymmetrien absichtlich nicht Verzerrungen (biases), weil dies implizieren würde, dass sie eine unverzerrte Formulierung kennen würden. Sie streben also nicht an, sogenannte 
Verzerrungen zu vermeiden sondern mit situativ unvermeidlichen Asymmetrien möglichst konstruktiv umzugehen.

Ich stelle diese Arbeit vor allem aufgrund ihres insgesamten Blicks auf Situationseinflüsse als gutes Beispiel vor. Ich möchte dennoch bezüglich der empirischen Durchführung etwas äußern, was einen anderen Punkt des Agential Realism betrifft. In Kapitel 5 habe ich herausgestellt, dass wir bei psychologischen Messungen zunächst herausarbeiten müssen, ob wir darin das Ergebnis nicht ausschließlich für das experimentelle Setting herstellen. So kann ich auch für die von Hegarty und Bruckmüller (2013) beschriebenen Studiendesigns fragen, ob die Einschätzungen als statushöher oder legitimer nicht erst durch die experimentelle Nachfrage danach hergestellt werden. Selbst wenn dem so wäre, hält ihre Argumentation meines Erachtens insgesamt trotzdem stand. Sozial relevant wird eine Auswirkung wie es legitim zu finden, dass Frauen weniger Geld verdienen als Männer dann, wenn darüber nachgedacht wird - egal durch wen oder was die Einschätzung evoziert wird. Es könnte also sein, dass die Legitimationseinschätzung erst entsteht, wenn irgendwas oder irgendwer danach fragt (d. h. die Konfigurationen dafür bestehen), und erst dann die Auswirkung geschieht - doch nur dann ist sie auch sozial relevant. Wenn sie nicht entsteht, wenn keine_r danach fragt, dann ist es auch irrelevant, weil niemand darüber nachdenkt. Dies kann analog zu folgendem Beispiel gesehen werden: Wenn wir nach dem Mond navigieren wollen, wäre es egal, wenn der Mond erst „entstehen würde, wenn wir hinsehen“ (vgl. Einsteins polemische Frage), denn sobald wir navigieren wollen, sehen wir hin und der Mond ist da.

Nach der Betrachtung der Verhältnisse von Agential Realism zu Queer Theory, der wissenschaftstheoretischen Position zur Methodenwahl und der Experimentalpsychologie mit klassischem Realismus zur Experimentalpsychologie mit Agential Realism, möchte ich nun auf die weitere Entwicklung einer ARqE blicken. Die Arbeit von Hegarty und Bruckmüller (2013) als Positivbeispiel für eine agential realist Herangehensweise anzuerkennen, kann zu der Frage führen, ob es also schon $A R$-Experimentalpsychologie gibt, die nur nicht dieses Label trägt. Ich spreche nun einige potentielle $A R$-Beispiele in Experimen- 
talpsychologie an; danach folgt eine Zusammenfassung meines Entwurfes der ARqE.

Die Tragweite der Kontextabhängigkeit eines jeden Phänomens wurde beispielsweise auch schon im Bereich der quantitativen social cognitionForschung diskutiert. In Kapitel 3.2.5, unter den Einschätzungen der Queerness der Experimentalpsychologie, wie auch in Kapitel 4.2.2, beim Entwurf der ARqE, habe ich die Ansätze der Situated Cognition kurz angesprochen, weil jene der queertheoretischen wie agential realist Forderung nachzukommen scheinen, den Kontext mit zum Phänomen zu denken. Nun will ich nach der ausführlichen Besprechung einer ARqE-Konzeption noch einmal einen Blick darauf werfen. Prinzipiell wird bei diesen Arbeiten auf Andy Clark referenziert, der 1997 mit "Being there: Putting brain, body and world together again" eine Art Grundlage für die Situated Cogniton geliefert zu haben scheint. Legt die Verständnisweise der Situated Cognition ein geeignetes Vorgehen für die ARqE nahe? Meine Antwort ist: Sie könnte, aber meistens geht sie nicht weit genug.

Eine neben der von Clark potentiell ebenfalls aufzunehmende Grundlagenarbeit für die Situated Cognition liefert die Philosophin Miriam Solomon (2007) mit ihrem Text "Situated Cognition" im Handbook of the Philosophy of Science. Philosophy of Psychology and Cognitive Science (Ed.: Paul Thagard). Sie referenziert auf zahlreiche frühere Einsichten (z. B. von Suchman über Clark und Haraway bis Latour und Hacking, vgl. Solomon, 2007, S. 413) um zu konstatieren, dass " $[R]$ epresentations of the world, learning, memory, planning, action and linguistic meaning are embedded in the environment, tools, social arrangements and configurations of the human body" (Solomon, 2007, S. 413). Außerdem hält sie fest: "[I]deologies that inform scientific creativity and scientific decision-making derive, in large part, from social variables including family psychodynamics, political orientation and societal position" (S. 413). Und weiter: "[S]cientists have situated knowledge practices that are constituted around local experimental successes and are dependent on particular tools, domains, historical contexts and forms of social organization" (413). Sie schließt ihren Artikel mit der Position: "[T]he most that scientists find is local regularities. I am arguing for a similar position in epistemology.” (S. 426) 
In Gänze scheint ihre Perspektive nicht von der Experimentalpsychologie aufgenommen worden zu sein, obwohl gleichzeitig die Kontextabhängigkeit für psychologische Forschung diskutiert wurde. Smith und Semin (2007) konstatieren in ihrem Text "Situated Social Cognition": "More progress might be made with a theoretical approach that makes interdependence and mutual constraint between person and context a central focus rather than a mere distraction from the inner representations" (S. 134). Allerdings erhält ihr Plädoyer - anders als das von Solomon - den Eindruck, dass es nur ein Phänomen bei Studienpartner_innen sei und keines, über das auch Forschende während ihres Erkenntnisprozesses nachdenken müssten (vgl. oben S. 115). Trotz Äußerungen wie "contexts pervasively influence social thought and action" (S. 135) und "[c]ommunication fundamentally shapes and even constitutes cognition, making cognition truly social" (S. 134), sowie der Einsicht, dass auch in Gegenstände, die wir verwenden, Wissen eingelassen ist (S. 134), wird an diesen Stellen nie auf "scientific thought and action" verwiesen oder festgestellt, dass "science truly social" ist bzw. auch unsere wissenschaftlichen Messapparate Gegenstände sind, in die Wissen eingelassen ist. Obwohl Smith und Semin (2007) eine Arbeit von Norenzayan und Schwarz (1999) zitieren, die die Bedeutung der oben genannten Punkte auf die Ebene der Einflüsse in Forschungsanstrangungen heben, gehen Smith und Semin insgesamt nicht soweit wie beispielsweise Gergen (2011), der die Verbundenheit von allem zum grundlegenden Ausgangspunkt nimmt.

Dies scheint sich im Feld durchzuziehen. Auch Yeh und Barsalou (2006) sprechen sich in "The Situated Nature of Concepts" dafür aus, alles unter Berücksichtigung des Kontextes zu betrachten: "The purpose of this review is to motivate the inclusion of background situations in future theories and in the research that accompanies them." (S. 359). Doch obwohl sie Situationseffekte als fundamental für Kognition erachten und als "present continuously during everyday activity" (S. 373), scheinen sie das nur auf Studienpartner_innen zu beziehen - die Bedeutung für Forschungsprozesse und deren Situiertheit wird mit keinem Wort diskutiert. Selbiges gilt auch für neuere Artikel, beispielsweise von Barsalou (2016) über "Situated Conceptualizations". 
Ähnlich verläuft es in einer Spezialausgabe des Journals Social Cognition mit dem Heft-Titel "Situated Social Cognition" (Social Cognition, 2013, Vol. 31, No. 2). Die Wichtigkeit, situationale Einflüsse einzuberechnen, wird mit zunehmender Evidenz begründet, dass auch ein größerer Kontext (statt beispielsweise nur die Stimulusgestalt) Ergebnisse beeinflusst. Das Ziel sind "findings that bear a broader ecological validity" (Jonas \& Cesario, 2013, S. 123), doch es wird nicht diskutiert, dass auch Forschende unter situationalen Einflüssen stehen. Am ehesten sind es wieder Schwarz und Kolleg_inn_en (Uskul, Oyserman, Schwarz, Lee \& Xu, 2013), die dieses Phänomen auch auf den Forschungsprozess beziehen. Sie konnten vorführen, dass neben der kulturellen Mentalität der antwortenden Personen auch die formalen Eigenschaften des Fragebogens (wie in meinem Kapitel 5) das Ergebnis mitbestimmen können. Es ist abzuwarten, inwieweit sich die Einsichten von Schwarz und Kolleg_inn_en durchsetzen, dass die Situiertheit auch für Forschungsergebnisse gilt.

Anschließend wäre relevant, wie dieser Einsicht begegnet würde. Bisher baut die Experimentalpsychologie diese Einsicht nicht grundlegend in ihre Forschungslogik ein. Vielmehr gelten ihre Bestrebungen weiterhin dem Versuch, Verzerrungen (von einer Wahrheit) zu verringern. So wirbt Brewer (2012) im Text zur "Theory Ladenness" angesichts dieser biases zu "reduce", "override incorrect" und "even out" (S. 328-329). Näher an Perspektiven der ARqE wäre der Ansatz der Situated Cognition, wenn er die Situiertheit auch auf den Forschungsprozess bezöge und dann nicht die Nivellierung dieser Situiertheit anstreben würde, sondern einen konstruktiven Umgang damit.

Ein anderes, womöglich vielversprechendes Feld für eine ARExperimentalpsychologie ist die Quantum Cognition-Forschung, die ich schon in Kapitel 4.2.3 bei der Entwicklung der ARqE erwähnt habe. Unter diesem Label gibt es eine wachsende Zahl von Forschungsarbeiten, Konferenzen und Kooperationen von verschiedenen Wissenschaftler_innen, die den Formalismus der Quantentheorie auf den Bereich der Kognition anwenden. Manche tun dies, wie in Kapitel 4.2.3 schon erwähnt, weil sie eine bessere Vorhersagekraft der quantentheoretischen Wahrscheinlichkeitsmodelle sehen, und andere, weil sie davon ausgehen, dass quantenmechanische Phänomene beim Funktionieren des Gehirns 
eine Rolle spielen. ${ }^{81}$ Die Forschenden scheinen sich dahingehend einig zu sein, dass die quantentheoretischen Formalismen sich für die Beschreibung von kognitiven Phänomenen, wie Entscheidungsfindung, Erinnerung oder Sprache, besser eignen als beispielsweise die Zugrundelegung Bool'scher Algebra: "The main argument for applying the formal apparatus of quantum theory to the domain of cognition has to do with the flexibility, instability, and context-dependency of natural concepts that manifest themselves as fleeting contents of conscious experience." (Quantum Cognition Network, 2016) Möglicherweise kann die Methodenanwendung der Quantum Cogniton-Forschung ein fruchtbares Gebiet für die $\mathrm{ARqE}$ und ihre eigene Methodenauseinandersetzung bieten (vgl. auch Kapitel 4.2.3). In solchen Potentialen zeigt sich der Bedarf differenzierter Auseinandersetzungen mit methodologischen Voraussetzungen, Einschränkungen und Möglichkeiten für ARqE. Abschließend möchte ich noch erwähnen, dass in der Theoretischen Psychologie (vgl. International Society for Theoretical Psychology (ISTP) und das Journal Theory \& Psychology) qua Interessensfokus immer wieder epistemologische und ontologische Grundlagen diskutiert werden (mitunter auch mit zu Barad vergleichbaren Teilperspektiven). Bisher scheinen diese Auseinandersetzungen jedoch keine grundlegende Änderung von Positionen in der Experimentalpsychologie bewirkt zu haben.

Für eine kurze Zusammenfassung der ARqE möchte ich festhalten, dass ich Herstellung der disziplinären Abgrenzung zwischen Psychologie und anderen Fächern wiederholt habe, während diese Grenze in Zukunft auch dekonstruiert werden könnte. Aktuell erachte ich diese Trennung als durch zahlreiche Konfigurationen (vgl. Kapitel 3) realisiert. Diese Konfigurationen stellen gleichermaßen Fachkonferenzen, Fachverbände, Fachzeitschriften etc. her. Deshalb erachte ich das vorläufige Wiederholen für sinnvoll. Außerdem erscheint mir ein Queeren der Disziplin günstiger aus der Disziplin heraus ${ }^{82}$, indem (wir) Experi-

81 Zwar werden die beiden Felder bei Wikipedia deutlich voneinander unterschieden, doch auf der Homepage des Networks Quantum Cognition finden sich sowohl die Verlinkung zu den Hypothesen des Quantum Minds wie auch die Vermischung der Forschenden mit unterschiedlichen Grün-

82 den für die Anwendung. Daher scheint mir die Trennung an dieser Stelle nicht so gravierend.

so wie Heinz-Jürgen Voß als Biolog_in die biologischen Konzeptionen anders wirkungsvoll dekonstruieren kann, als beispielsweise ich das könnte. 
mentalpsycholog_innen zunächst selbst (unsere) Konzeptionen von Ojektivität, von Test-Ergebnissen, von Interpretationen, von Frageweisen etc. queeren. Doch aus Agential Realist Queer(ing)-Perspektive kann auch erstrebenswert sein, die disziplinären Grenzen überhaupt zu dekonstruieren, wonach dann eine Bezeichnung als Psychologie abzulegen wäre. Dann wäre für die Zukunft womöglich eine Agential Realist Queer(ing) Science angestrebt, wobei ich in dieser Arbeit auf (situativ) praktikable Differenzierungsmöglichkeiten innerhalb einer solchen (z. B. welches Fach man dann studieren würde) nicht weiter eingehen werde.

Was kann diese von mir skizzierte ARqE Forschenden bieten? Experimentalpsycholog_inn_en, die (weiter) nach Nähe zur Wahrheit streben, könnte interessieren, wenn ARqE-Vorgehen genauere Vorhersagen liefert. Dies müsste sich in Zukunft zeigen. Jene Experimentalpsycholog_inn_en, die einerseits davon fasziniert sind, dass menschliches Verhalten manchmal vorhergesagt werden kann, und andererseits Menschen nicht normieren wollen, wird interessieren, dass ARqE relata-in-relations sieht. Sie werden womöglich damit arbeiten, dass Wissenschaft situativ „funktioniert" ${ }^{83}$, aber dies nicht bedeutet, menschenunabhängige Fakten über die Natur zu entdecken. Queertheoretiker_innen kann interessieren, dass Experimente nützlich sein können und nicht zwingend normierend sind; dass es vielmehr darauf ankommt, wie Experimente gemacht und welche Schlüsse aus ihren Ergbenissen gezogen werden. Dass Wissenschaft funktioniert, können wir - so meine Hoffnung - auch situiert und queertheoretisch positiv benutzen. Agential Realist_innen halten es vermutlich für selbstverständlich, dass ihre Perspektiven auch für die Psychologie und ihre Phänomene wichtig sind. Für sie ist möglicherweise interessant zu sehen, wie sich diese Bedeutung für die Psychologie übersetzen lassen könnte. Letzteres habe ich mit der Entwicklung von ARqE in dieser Arbeit darzustellen versucht.

Eventuell ist es diese Unterschiedlichkeit der Perspektiven darauf, was interessiert, bzw. auch, was als Neuerung oder Markantes einer Position verstanden wird, die dazu führt, jeweils verschiedene Aspekte zu betonen. Für den Blick auf eine Experimentalpsychologie, die zum Großteil davon ausgeht, dass Materie 
Phänomene determiniert, scheint mir die Betonung der Mitwirkungsmacht der Forschenden wichtig. Deshalb habe ich dies in der vorliegenden Arbeit mehr in den Vordergrund gestellt als die Grenzen, die durch materielle Konfigurationen situativ wirken können. Für andere Perspektiven kann der entscheidende Beitrag des Agential Realism sein, dass auch Materie eine (wenn auch nicht im menschlichen Sinne) Mitwirkungsmacht haben soll. Für meine Argumentation ist die agential realist-Perspektive auf materiell-diskursive Konfigurationen der fruchtbare Punkt - welche entsprechend des Konzeptes der Intra-aktion nicht als Additionen zweier Bereiche verstanden werden, sondern als untrennbar verschränkte -, um über die Mitwirkung von Forschenden bei sogenannten naturwissenschaftlichen Experimenten zu sprechen.

Gegenüber klassischen Forschungsfragen nach Beschaffenheiten ist es eine Neuerung der ARqE zu fragen, welche Realisierungsmöglichkeiten es noch gibt. Wenn es mehrere Gestalten eines Phänomens gibt, können situativ Entscheidungen nötig sein, welche Gestalt zu bevorzugen ist. Im Falle von Licht scheint es für die verschiedenen Gestalten von Licht (Teilchen, Welle) verschiedene Nutznießer zu geben. Das Chlorophyll in Pflanzen scheint die Teilchen zu verwenden, während ein Fensterkristall, der kleine Regenbögen in ein Zimmer wirft, die Wellen verwendet. Eine Kultur, die sich auf Distinktion verlässt, kann die Stereotypisierungsfähigkeit von Menschen verwenden. Eine Kultur, die sich auf Communion ${ }^{84}$ verlässt, würde vielleicht andere menschliche Fähigkeiten verwenden. Zu sagen, der Mensch stereotypisiere unweigerlich, ist entsprechend etwas anderes, als zu sagen, der Mensch hat die Fähigkeit zu stereotypisieren. Solche Entscheidungen werden zweifelsohne immer wieder getroffen. Als problematisch erachte ich jedoch, wenn diese nicht als Entscheidungen wahrgenommen und benannt, sondern die resultierenden Ergebnisse als neutrale Fakten erachtet werden. Thomas Teo $(2008,2010)$ diskutiert in diesem Zusammenhang besonders die Fälle, in denen bei der Interpretation von Daten bestimmte Gruppen als unterlegen dargestellt werden. Dies ist nicht per se Ergebnis der Daten, sondern resultiert aus Entscheidungen von Forschenden, weshalb Teo es (in Erweiterung des Konzeptes epistemischer Gewalt) epistemological violence

84 i.S.v. Gemeinschaft, Verbundenheit, Teilhabe 
(Teo, 2008) nennt. Es könnte interessant sein, sich damit zu befassen, welche Kriterien für solche Entscheidungsfindungen bereits herangezogen werden. Gerade für eine ARqE, die ihren Forschenden (Mit-)Verantwortung für die Gestalten ihrer Ergebnisse zuschreibt, ist es zudem besonder wichtig, zukünftig Kriterien für diese Entscheidungen zu entwickeln.

$\mathrm{Da}$ in solche Kriterien auch einfließt, wie wir leben wollen, müssen solche Aushandlungsprozesse nicht nur innerhalb einer wissenschaftlichen Disziplin, sondern gesamtgesellschaftlich geführt werden. Von Vorschlägen für einen solchen Prozess sehe ich in dieser Arbeit ab, da dies hochkomplexe Aushandlungsprozesse von verschiedenen Werten erfordert. Man stelle sich nur die noch vergleichsweise simple Konstellation vor, dass einige Menschen wollen, dass sogenannte Männer und sogenannte Frauen differenziert werden und andere wollen dies nicht. Wir können allein durch Konfiguration unserer Messapparate Verschiedenheit oder Gleichheit herstellen - welche Gestalt ist erstrebenswerter? Queertheoretische Analysen können für einige solcher Fragen bereits Antworten liefern und Werte wie Begründungen für weitere Entscheidungsfindungen bereitstellen, doch nicht immer. Vor dem Angang dieser Herausforderungen ist es jedoch wichtig, dass die Macht von Forschenden wahrgenommen wird und nicht weiter unproblematisiert epistemologische Gewalt ausgeübt werden kann.

Wenn die leitende Frage für Forschung ist, wie wir leben wollen, und wenn Möglichkeiten statt Gegebenheiten gesucht werden, dann müssen auch (neue) Kriterien entwickelt werden, wie die Interessantheit einer Forschungsfrage beurteilt wird. Bisher gilt als einzige Rechtfertigung, sich einer Forschungsfrage zu widmen, dass man bisher zu wenig über das Thema weiß (wobei immer einseitges Wissen für nur manche Bevölkerungsgruppen produziert wurde). Wenn nun aber die Welt nicht etwas wie einem Uhrwerk entspricht, dessen Mechanik wir entdecken können, sondern vielmehr verschiedenste Möglichkeitsräume bereithält (je nachdem welche Konfiguration wir-in-relations situativ realisieren), kann die Suche dann weiterhin - wie im Humboldt'schen Ideal vom zweckfreien Wissenserwerb - allen möglichen Realisierungsformen gelten? 
Bislang habe ich mich darauf zurückgezogen, dass Forschungsfragen begründet werden müssen (vgl. Kapitel 5.2). Dies soll Reflektionen provozieren, warum man Forschungsfragen in einer bestimmten Art und Weise formuliert und nicht anders. Zweifelsohne ist auch dieser Frage, wie die Güte von Begründungen für Formulierungsweisen von Forschungsfragen aus ARqE-Perspektive beurteilt werden, noch nachzugehen. Ebenso habe ich in Kapitel 5 den Grad der Bewusstheit für eine bestimmte Problematik angesprochen, welcher häufig schwer zu beurteilen ist bzw. je nach Standpunkt auch sehr verschieden beurteilt wird. Wie schwer wiegt beispielsweise die queertheoretisch kritisch gesehene Reifikation von Geschlechterbinarität in welcher Konstellation und wie gut ist die Problematik analysiert? Für solche Fragen kann es keinen universalen Kriterienkatalog geben. Dennoch halte ich Diskussionen über die Abwägung von Forschungsanstrengungen, die unsere Welt mitgestalten (statt sie nur abzubilden), für unausweichlich.

Die Problematik der gesellschaftlichen Verantwortung von Wissenschaft trennt die klassisch betriebene Psychologie von kritisch, feministisch und queertheoretisch informierten Wissenschaften. Für die feministisch und queertheoretisch fundierten Gender Studies erachte ich den Eingangs von Steffens und Ebert (2010) festgestellten Graben gegenüber „dem internationalen Establishment in der heutigen akademischen Psychologie“ (vgl. Steffens \& Ebert, 2010, S. 194) wie diese als sehr groß. Sie schreiben: „Es ist jedoch zu beachten, dass eine gemeinsame Basis noch zu schaffen ist: Welche Methoden gelten als anerkannt, um Erkenntnisfortschritte zu erzielen? Welche Konzepte werden wie verwendet und haben welche Implikationen? (...) Auf welcher Abstraktionsebene forschen wir?" (Steffens \& Ebert, 2014, S. 203, siehe auch oben S. 2).

Für eine Disziplin, die wie die Experimentalpsychologie quantitativ vorgehen will, halte ich den Agential Realism für eine „gemeinsame Basis“ zur Überwindung dieses Grabens (vgl. Steffens \& Ebert, 2014, S. 203). So bietet die $\mathrm{ARqE}$ schon zahlreiche Weiterentwicklungen und Lösungen an. In der ARqE ist gefordert, dass jede Forschungsanstrengung selbst explizieren und begründen muss, auf welcher Abstraktionsebene sie forscht. Ebenso muss die (und sei es temporäre und lokale) Festlegung von Konzepten expliziert und begründet wer- 
den. Die Implikationen dieser Festlegungen sind dann wiederum selbst kontextabhängig. Die Antwort der ARqE auf die Frage nach der Anerkennung von Methoden - unabhängig davon, dass sich Anerkennung auch erst in Zukunft zeigen wird - lässt sich aufspalten in Anerkennung von ,(1) Methoden zur Datenerhebung, (2) Methoden zur Datenauswertung, (3) Methoden zur Hypothesenbildung und (4) Methoden zum Hypothesentest.“ (vgl. Lauth \& Sareiter, 2005, S. 15) Hier ist zweifelsohne noch (Entwicklungs- und Umgestaltungs-)Arbeit zu leisten. Die Güte einer eingesetzten Methode zeigt sich gemäß ARqE nicht mehr in Reliabilität, Validität und Objektivität im klassischen Sinne. Vielmehr müssen die Methoden eine vorher spezifizierte Funktion erfüllen (und Forschende Barads Konzeption von Objektivität umsetzen). Wenn wir weiterhin davon ausgehen, dass Methoden mitkreieren, dann müssen auch die in dieser Arbeit entwickelten Gütekriterien eingelöst werden (und sei es graduell):

- $\quad$ Es wird Bewusstheit für die und reflektierter Umgang mit den Relationen von Relata gefordert.

- Es ist möglichst eindeutig verstehbar über alle mitwirkenden Intraaktionen (auch Einstellungen und Überzeugungen der Forschenden) zu kommunizieren.

- Kausalrelationen werden nur in ihrer Bedingungsabhängigkeit beschrieben.

- Vorannahmen und Perspektiven von Forschenden sind zu nennen; der Diskurs, in dem eine Beschreibung geschieht, ist zu explizieren.

- $\quad$ Forschende müssen selbstreflexiv und begründet mit ihrer Forschung und deren Wirkungen umgehen (nicht in direkt politischem Sinn, sondern dahingehend, welche Gestalt sie mitgestalten).

Eine versierte Weiterentwicklung oder passendere Formulierung dieser Kriterien sowie die Neuentwicklung weiterer Kriterien für eine gute ARqE halte ich für äußerst wünschenswert. Diese Arbeit hatte zum Ziel, den Anfang der Synthese einer queer(end)en Experimentalpsychologie zu machen. Mit dieser Arbeit erhebe ich den Anspruch, konkrete Anwendungsmöglichkeiten der metatheoretischen Positionen zu liefern, auch wenn direkte Forschungsarbeiten bisher 
von anderen Kolleg_inn_en geliefert wurden. Konkrete Erinnerungen für zukünftige Forschungsanstrengungen sind:

- Keine Feststellung über Phänomene und keine Begründung von Forschungsfragen kann lauten, dass etwas so sei und lediglich sogenannte Fakten der Welt entdeckt werden sollen - dies kann lediglich über lokal und temporär eingeschränkte, situative Zusammenhänge gesagt werden, die dennoch einen Realisierungsraum, und nicht nur eine einzelne Realisierungsmöglichkeit bieten.

- Wir erforschen die relations der relata-within-relations.

- Wir forschen nicht nach Erklärungen auf der Ebene von stabilen Eigenschaften von Entitäten, sondern auf der Ebene von spezifischen Konfigurationen eines Phänomens.

- $\quad$ Als Forschende erkennen wir an, dass Abweichung ein relationaler Begriff dazu ist, was wir vorher als Norm definiert haben; von einem anderen Standpunkt aus wäre beides möglicherweise vertauscht. Wir können mit Achsenkreuzen arbeiten, solange explizit ist, dass diese nicht in einer Natur der Sache liegen, sondern durch Konfigurationen hergestellt sind.

- Wir erkunden den Möglichkeitsraum.

- Wir erkennen einen Realisierungsraum, statt ein Resultat plus zufälligen Fehler.

- Entsprechend Augoustinos und Kolleg_inn_en wiederholen wir nicht den früheren Fehler: "[We do not] exempt [ourselves] and [our] theories from the constructivist principles [we] apply to the subjects of [our] research and theorizing." (vgl. Augoustinos et al., 2014, S. 20)

- Nach einer empirischen Realisierungserprobung (z. B. „Menschen stereotypisieren") erachten wir dies als menschliche Möglichkeit und nicht als Unweigerlichkeit (z. B. kann dann eine Folgefrage lauten: „Wann stereotypisieren Menschen nicht?")

Analogien wie Zahnräder im Kopf (vgl. u. a. Titelbild von Kashima et al., 2008, siehe auch Kap. 3.1.3) verbieten sich entsprechend. Passender wäre ein Bild wie das der Hände MC Eschers, die sich gegenseitig zeichnen. Die Agential 
Realist Queer(ing) Psychology geht von der gleichzeitigen Existenz von Bedingungen und Agency aus.

Judith Butler sagt über ihr Buch "Notes toward a performative theory of assembly": "The thesis of this book is that none of us acts without the conditions to act, even though sometimes we must act to install and preserve those very conditions." (Butler, 2015, S. 16) Was in diesem Satz paradox klingen mag, spricht Butler im direkt folgenden Satz an - es ist nur in einer klassisch realistischen, deterministischen Perspektive scheinbar paradox. Der Satz exemplifiziert die Perspektive der gleichzeitigen Anerkennung von Bedingungen und Agency. Darin ist Butlers Einsicht meines Erachtens deckungsgleich mit Barads und entgegen der Annahme, dass stabile Entitäten nach Logik von Newton'scher Mechanik ein Uhrwerk bilden. Psychologische Forschung kann die Logik Newton'scher Mechanik verlassen und das Agential Realist queer(end)e InBeziehung-Setzen einnehmen; queer(end)e Experimentalpsychologie ist dann kein Widerspruch. Die relata als in-relations zu sehen, zwingt uns Forschende dazu, nicht nach individuellen Essenzen zu suchen, sondern die jeweilige Relationalität ${ }^{85} \mathrm{zu}$ finden. Die Existenz von agential cuts bestätigt auch, dass wir situative Grenzen finden und nicht alles beliebig hergestellt werden kann. Gleichzeitig bedeuten agential cuts auch agency, (Mit-) Wirkungsmacht, und dass wir Neugestaltungen mitbewirken können - in relations. Forschung kann helfen, Möglichkeits- und Realisierungsräume anstelle einschränkender irrelationaler Eigenschaftsaussagen zu entdecken. Damit ist Forschung ein Werkzeug, um die Frage zu explorieren „Wie geht es außerdem?“ So wie auch queerende Menschen, ohne andere einzuschränken, immer wieder die Möglichkeiten des Lebens ausprobier(t)en und durch das Lossagen von essentialisierenden Festschreibungen - wie angeblich dies sei und jenes nur so funktioniere - neue Realisierungen von Leben entdeck(t)en, welche weniger Einschränkungen und cuts, sondern mehr Freiheiten mitbringen, sowie Weiter-

85 Es wird kein Zufall sein, dass „etwas zu relativieren“ im Deutschen die negative Konnotation trägt, etwas abzuschwächen oder einzuschränken (vgl. u. a. Wiktionary (2016): „die Bedeutung von etwas abschwächen, indem man es zu einer anderen Sache in Beziehung setzt" und Dudenredaktion (2016): ,zu etwas anderem in Beziehung setzen und dadurch in seinem Wert o. Ä. einschränken“). 


\section{entwicklung anstoßen. Entscheidend ist also die Frage: Welche Welt ist noch möglich? Verbunden mit der Frage: Welche Welt wollen wir?}

Open Access Dieses Kapitel wird unter der Creative Commons Namensnennung 4.0 International Lizenz (http://creativecommons.org/licenses/by/4.0/deed.de) veröffentlicht, welche die Nutzung, Vervielfältigung, Bearbeitung, Verbreitung und Wiedergabe in jeglichem Medium und Format erlaubt, sofern Sie den/die ursprünglichen Autor(en) und die Quelle ordnungsgemäß nennen, einen Link zur Creative Commons Lizenz beifügen und angeben, ob Änderungen vorgenommen wurden.

Die in diesem Kapitel enthaltenen Bilder und sonstiges Drittmaterial unterliegen ebenfalls der genannten Creative Commons Lizenz, sofern sich aus der Abbildungslegende nichts anderes ergibt. Sofern das betreffende Material nicht unter der genannten Creative Commons Lizenz steht und die betreffende Handlung nicht nach gesetzlichen Vorschriften erlaubt ist, ist für die oben aufgeführten Weiterverwendungen des Materials die Einwilligung des jeweiligen Rechteinhabers einzuholen. 